\title{
Gravitational-Wave Emission from Rotating Gravitational Collapse in three Dimensions
}

\author{
L. Baiotti ${ }^{1}$, I. Hawke ${ }^{2,4}$, L. Rezzolla ${ }^{1,3}$, and E. Schnetter ${ }^{2}$ \\ ${ }^{1}$ SISSA, International School for Advanced Studies and INFN, Via Beirut 2, 34014 Trieste, Italy \\ ${ }^{2}$ Max-Planck-Institut für Gravitationsphysik, Albert-Einstein-Institut, 14476 Golm, Germany \\ ${ }^{3}$ Department of Physics, Louisiana State University, Baton Rouge, LA 70803 USA and \\ ${ }^{4}$ School of Mathematics, University of Southampton, Southampton SO17 1BJ, UK
}

(Dated: August 29, 2018)

\begin{abstract}
We present the first calculation of gravitational wave emission produced in the gravitational collapse of uniformly rotating neutron stars to black holes in fully three-dimensional simulations. The initial stellar models are relativistic polytropes which are dynamically unstable and with angular velocities ranging from slow rotation to the mass-shedding limit. An essential aspect of these simulations is the use of progressive mesh-refinement techniques which allow to move the outer boundaries of the computational domain to regions where gravitational radiation attains its asymptotic form. The waveforms have been extracted using a gauge-invariant approach in which the numerical spacetime is matched with the non-spherical perturbations of a Schwarzschild spacetime. Overall, the results indicate that the waveforms have features related to the properties of the initial stellar models (in terms of their $w$-mode oscillations) and of the newly produced rotating black holes (in terms of their quasi-normal modes). While our waveforms are in good qualitative agreement with those computed by Stark and Piran in two-dimensional simulations [1], our amplitudes are about one order of magnitude smaller and this difference is mostly likely due to our less severe pressure reduction. For a neutron star rotating uniformly near mass-shedding and collapsing at $10 \mathrm{kpc}$, the signal-to-noise ratio computed uniquely from the burst is $S / N \sim 0.25$, but this grows to be $S / N \lesssim 4$ in the case of LIGO II.

PACS numbers: 04.25.Dm, 04.30.Db, 04.70.Bw, 95.30.Lz, 97.60.Jd
\end{abstract}

The study of the gravitational collapse of rotating stars to black holes is a cornerstone of any theory of gravity and a long standing problem in general relativity. Over the years, numerous approaches have been applied and several different techniques developed to tackle this problem which is not just academic. Indeed, important issues in relativistic astrophysics awaiting clarification, such as the mechanism responsible for $\gamma$-ray bursts, may be unveiled with a more detailed understanding of the physics of gravitational collapse in rotating and magnetized stars. Furthermore, the study of gravitational collapse will provide the waveforms and the energetics of one of the most important sources of gravitational radiation.

In the absence of symmetries, no analytic solutions are known that describe the gravitational collapse to a black hole and it is therefore through numerical relativity simulations that one can hope to improve our knowledge of this process under realistic conditions. However, this is not an easy task and the modelling of black hole spacetimes with collapsing matter-sources in multidimensions is one of the most formidable efforts of numerical relativity. This is due both to the inherent difficulties and complexities of the system of equations which is to be solved (i.e. the Einstein equations coupled to the relativistic hydrodynamics equations) and to the vast computational resources needed in three-dimensional (3D) evolutions.

In addition to the technical difficulties due to the accurate treatment of the hydrodynamics involved in the collapse, the precise calculation of the gravitational radiation emitted in the process is particularly challenging as the energy released in gravitational waves is much smaller than the total rest-mass energy of the system. Indications of the difficulties inherent to the problem of calculating the gravitational-wave emis- sion in rotating gravitational collapse have emerged in the first and only work on this, which dates back to almost 20 years ago [1]. In 1985, in fact, in a landmark work in numerical relativity, Stark and Piran used their axisymmetric generalrelativistic code to evolve rotating configurations and to compute the gravitational radiation produced by their collapse to black holes. The results referred to initial configurations consisting of polytropic stars which underwent collapse after the pressure was reduced by a factor ranging from $60 \%$ up to $99 \%$ for the rapidly rotating models. The initial data effectively consisted of spherically-symmetric solutions with a uniform rotation simply "added" on. Not being stationary solutions of the Einstein equations, these stars could reach dimensionless spins up to $a \equiv J / M^{2}=0.94$, with $J$ and $M$ the angular momentum and mass of the star, respectively.

Overall, their investigation revealed that while the nature of the collapse depended on the parameter $a$, the form of the waves remained roughly the same over the entire range of the values of $a$, with the amplitude increasing with $a$. Particularly important was the evidence that, despite the complex dynamics of the matter during the collapse, the gravitational-wave emission could essentially be related to the oscillations of a perturbed black hole spacetime.

In recent years many studies have extended to three spatial dimensions the investigation of gravitational collapse to black holes [2, 3, 4]. Despite the improvements in the evolution of the hydrodynamics and of the spacetime achieved by these simulations, none of them has addressed the problem of the gravitational-wave emission. The reason for this is to be found in the small amplitude of the signal, often below the truncation error of the 3D simulations, but most importantly in the fact that all of the above calculations made use of Cartesian 
grids with uniform spacing. With the computational resources currently available, this choice places the outer boundaries too close to the source to detect gravitational radiation.

Using a recently developed code for the solution of the Einstein equations in non-vacuum spacetimes, the Whisky code, we have investigated the collapse of rapidly rotating relativistic stars to Kerr black holes [5]. An important aspect of these simulations is the detailed study of the geometrical and dynamical properties of both the apparent and event horizons, allowing for the determination of both the mass and angular momentum of the black hole with an accuracy unprecedented for a 3D simulation. In turn, these measures have set upper limits on the energy and angular momentum lost during the collapse in the form of gravitational radiation, the first such estimates coming from 3D calculations.

However, as in previous works (e.g. refs. [2, 3, 4]), the simulations reported in [5] made use of numerical grids with uniform spacing and thus with outer boundaries very close to the initial position of the stellar surface. With these restrictions, the gravitational radiation extracted does not provide interesting information besides the obvious change in the quadrupole moment of the background spacetime. As we will show below, the use of progressive mesh-refinement (PMR) techniques has removed these restrictions, enabling us to place the outer boundaries of the computational domain at very large distances from the collapsing star. This conceptually simple but technologically challenging improvement has two important physical consequences. Firstly, it reduces the influence of inaccurate boundary conditions at the outer boundaries of the domain whilst retaining the required accuracy in the region where the black hole forms. Secondly, it allows the wavezone to be included in the computational domain and thus to extract the gravitational waves produced in the collapse.

In practice, we have adopted a Berger-Oliger prescription for the refinement of meshes on different levels [6] and used the numerical infrastructure described in [7]. In addition to this, we have also implemented a simplified form of adaptivity in which new refined levels are added at predefined positions during the evolution. More specifically, given an initial stellar model of mass $M$ and equatorial coordinate radius $R$, our initial grid consists of four levels of refinement, with the innermost one covering the star with a typical resolution of $\Delta x \sim$ $0.17 \mathrm{M}$ and with the outermost having a typical resolution of $\Delta x \sim 1.38 M$ and extending up to $\sim 82.5 M \simeq 20.9 R$. As the collapse proceeds and the star occupies smaller portions of the computational domain, three more refined levels are added one by one, nested in the four original ones. By the time the simulation is terminated at $\sim 81.5 \mathrm{M}$, the finest typical spatial resolution is $\Delta x \sim 0.02 M$. A detailed discussion of the grid and of its evolution will be given in [8].

The initial data for our simulations is the same described in [5] and basically consists of axisymmetric rotating relativistic stars, calculated as equilibrium solutions of the Einstein equations in a compactified domain and in polar coordinates. For a direct comparison with the results in [1] and because no shock is observed during the collapse, the stars are modelled with a polytropic equation of state (EOS) $p=K \rho^{\Gamma}$, with $\Gamma=2$ and with the polytropic constant which is initially $K_{\mathrm{ID}}=100$. Once secularly unstable solutions are found along sequences of fixed angular momentum or fixed rest-mass, we consider initial models that have the same axis ratios but slightly larger central energy densities and are dynamically unstable. Hereafter we will restrict the discussion to the collapse of the most rapidly rotating dynamically unstable model, namely model D4 in [5], which represents a star of mass $M=1.861 M_{\odot}$, circumferential equatorial radius $R_{e}=14.25 \mathrm{~km}$ and rotating close to the mass-shedding limit with $a=0.54$ ( $c f$. Table I of [5]). The discussion of the emission from stellar models rotating at smaller velocities will be presented in [8].

Although model D4 is dynamically unstable, it is very close to a stationary solution and we trigger its collapse by reducing the pressure by $\lesssim 2 \%$, with a change in the value of the polytropic constant. After the perturbation is introduced, the constraint equations are again solved to enforce the constraint violation to be at the truncation-error level. As a contrast, in ref. [1], rapid rotation was added to a spherically symmetric solution and the large pressure depletion inevitably produced a dependence of the results on the amount of pressure reduction. Hereafter we will concentrate on the gravitational-wave emission, but a discussion of the dynamics of matter and trapped surfaces can be found in [5].

While several different methods are possible for the extraction of the gravitational-radiation content in numerical spacetimes, we have adopted a gauge-invariant approach in which the spacetime is matched with the non-spherical perturbations of a Schwarzschild black hole (see refs. [9, 10, 11] for applications to Cartesian coordinates grids). In practice, a set of "observers" is placed on 2-spheres of fixed coordinate radius $r_{\text {ex }}$, where they extract the gauge-invariant, odd $Q_{\ell m}^{(o)}$ and even-parity $\Psi_{\ell m}^{(\mathrm{e})}$ metric perturbations [12]. Here $\ell, m$ are the indices of the angular decomposition and we usually compute modes up to $\ell=5$ with $m=0$; modes with $m \neq 0$ are essentially zero because of the high degree of axisymmetry in the collapse. Validations of this approach in 3D vacuum spacetimes can be found in refs. [10, 13, 14], while its use with matter sources has first been reported in [15].

Although the position of such observers is arbitrary and the information they record must be the same for waves extracted in the wave-zone, we place our observers between $40 M$ and $50 \mathrm{M}$ from the centre of the grid so as to maximize the length of the extracted waveform. We note that while a similar choice was made in ref. [1], it still provides only an approximate description of what would be observed at spatial infinity.

Using the odd and even-parity perturbations $Q_{\ell m}^{\times}=\lambda Q_{\ell m}^{(\mathrm{o})}$ and $Q_{\ell m}^{+}=\lambda \Psi_{\ell m}^{(\mathrm{e})}$, where $\lambda \equiv \sqrt{2(\ell+2) ! /(\ell-2) !}$, we report in Fig. 11 the lowest-order multipoles for $Q_{\ell m}^{+}$with the offset produced by the stellar quadrupole removed [8]. The left panel, in particular, refers to the $\ell=2$ mode as extracted by four different observers at increasing distances and expressed in retarded time. The right panel instead refers to the $\ell=4$ mode, with the inset giving a comparison between the two 

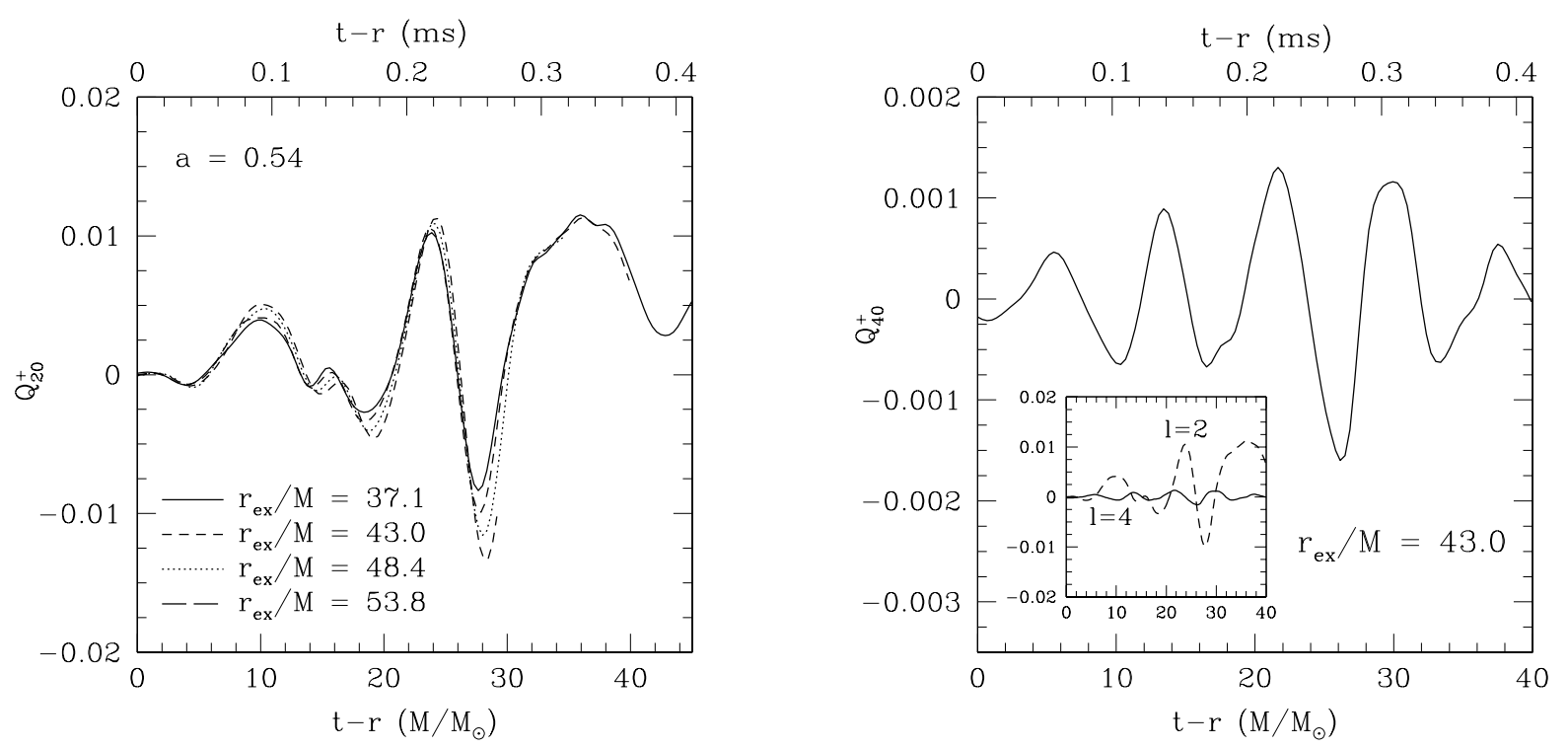

FIG. 1: The left panel shows the $\ell=2$, even-parity perturbation as extracted by observers at different positions $r_{\text {ex }}$ expressed in retarded time. The right panel refers to the $\ell=4$ mode, with the inset offering a comparison in the amplitudes of the two modes.

modes and showing that the gravitational-wave signal is essentially quadrupolar, with the $\ell=2$ mode being about an order of magnitude larger than the $\ell=4$ mode.

The very good overlap of the waveforms measured at different positions is important evidence that the extraction has been performed in the wave-zone, since the invariance under a retarded-time scaling is a property of the solutions of a wave equation. The overlap disappears if the outer boundary is too close or when the waves are extracted at smaller radii. A similar overlap is seen also for the $\ell=4$ mode (not shown).

Another indication that the waveforms in Fig. 1 are an accurate description of the gravitational radiation produced by the collapse comes by analysing their power spectra. The collapse, in fact, can be viewed as the rapid transition between the spacetime of the initial equilibrium star and the spacetime of the produced rotating black hole. It is natural to expect, therefore, that the waveforms produced in this process will reflect the basic properties of both spacetimes and in particular the fundamental frequencies of oscillation. We validate this in Fig. 2 where we show the power spectral densities (PSD) of the waveforms of the metric perturbations $Q_{20}^{+}$and $Q_{40}^{+}$reported in Fig. 11(the units on the $y$-axis are arbitrary). The upper panel of Fig. 2 in particular, shows the PSD of $Q_{20}^{+}$and compares it with the frequencies of the $\ell=2, m=0$ quasi-normal mode (QNM) of a Kerr black hole with $M=1.861 M_{\odot}$ and $a=0.6$ [16] (dashed line at 6.7 $\mathrm{kHz})$ as well as with the first $w_{\mathrm{II}}$ "interface" mode for a typical compact star with $M=1.27 M_{\odot}$ and $R=8.86 \mathrm{~km}$ (dotted line at $8.8 \mathrm{kHz}$ [17]. Similarly, the lower panel of Fig. 2] shows the PSD of $Q_{40}^{+}$comparing it with the $\ell=4, m=0$ QNM of a Schwarzschild black hole [17] (dashed line at 14.0 $\mathrm{kHz}$ ) and the first $w_{1}$ "curvature" mode [17] (dotted line at $12.8 \mathrm{kHz}$ ) [18]. It is quite apparent that both peaks in the PSDs are rather far from the maximum sensitivity area of modern interferometric and bar detectors.
Although the waveforms have very short duration with very broad PSDs, Fig. 2 shows that these have strong and narrow peaks (a similar behaviour can be shown to be present also for the odd-parity modes [8]). Indeed, the excellent agreement between the position of these peaks and the fundamental frequencies of the vacuum and non-vacuum spacetimes is an important confirmation of the robustness of the results obtained.

Using the extracted gauge-invariant quantities it is also possible to calculate the transverse traceless (TT) gravitationalwave amplitudes in the two polarizations $h_{+}$and $h_{\times}$as

$$
h_{+}-\mathrm{i} h_{\times}=\frac{1}{2 r} \sum_{\ell, m}\left(Q_{\ell m}^{+}-\mathrm{i} \int_{-\infty}^{t} Q_{\ell m}^{\times}\left(t^{\prime}\right) d t^{\prime}\right)_{-2} Y^{\ell m}
$$

where ${ }_{-2} Y^{\ell m}$ is the $s=-2$ spin-weighted spherical harmonic. Because of the small amplitude of higher-order modes, the TT wave amplitudes can be simply expressed as $h_{+} \simeq h_{+}\left(Q_{20}^{+}, Q_{40}^{+}\right)$and $h_{\times} \simeq h_{\times}\left(Q_{30}^{\times}, Q_{50}^{\times}\right)$, where $Q_{30}^{\times} \gg Q_{50}^{\times}$. Their waveforms are shown in Fig. 3 for the detector at $r_{\mathrm{ex}}=37.1 \mathrm{M}$ and for two different inclination angles [8]. Note that the amplitudes in the cross polarization are about one order of magnitude smaller than those in the plus polarization, with the maximum amplitudes in a ratio $\left|(r / M) h_{\times}\right|_{\max } /\left|(r / M) h_{+}\right|_{\max } \simeq 0.06$. Here, the oddparity perturbations, which are zero in spacetime with axial and equatorial symmetries, are just the result of the coupling, induced by the rotation, with the even-parity perturbations.

A precise comparison of the amplitudes in Fig. 3 with the corresponding ones calculated in [1] is made difficult by the differences in the choice of initial data and, in particular, by the impossibility of reaching $a \gtrsim 0.54$ when modelling consistently stationary polytropes in uniform rotation. However, when interpolating the results in [1] for the relevant values of $a$, we find a very good agreement in the form of the waves, but also that our estimates are about one order of magnitude 


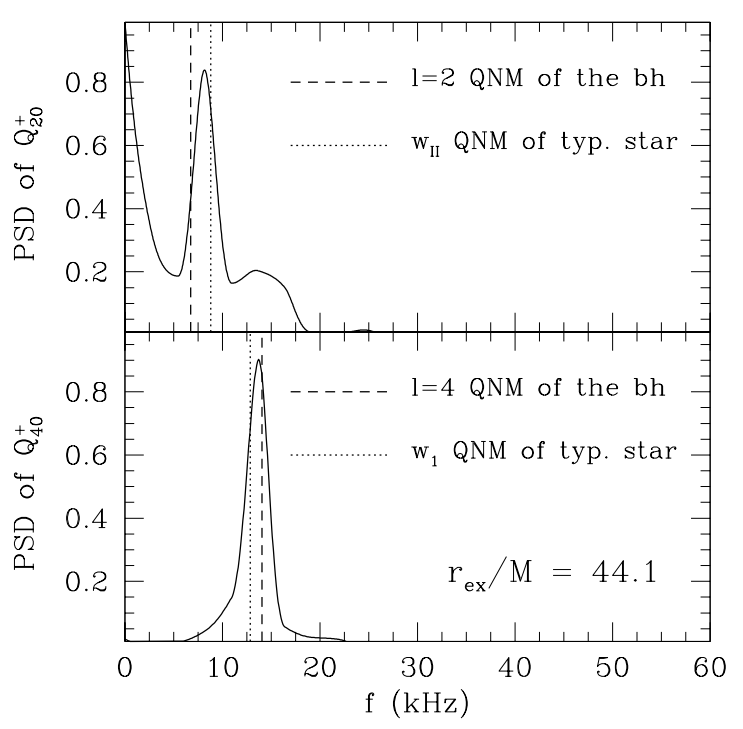

FIG. 2: Power spectra of the waveforms reported in Fig. 11 The dashed vertical lines indicate the frequencies of the QNMs of a black hole, while the dotted ones the $w_{1}$ and $w_{\text {II }}$ modes of a typical star.

smaller, with $\left|(r / M) h_{+}\right|_{\max } \simeq 0.00225$. Furthermore, we observe the amplitude of the gravitational waves to increase with the pressure reduction, thus suggesting that the origin of the difference is related mainly to this [8].

Following ref. [19] and considering the optimal sensitivity of VIRGO for the burst signal only, we set an upper limit for the characteristic amplitude produced in the collapse of a rapidly and uniformly rotating polytropic star at $10 \mathrm{kpc}$ to be $h_{c}=5.77 \times 10^{-22}\left(M / M_{\odot}\right)$ at a characteristic frequency $f_{c}=931 \mathrm{~Hz}$. In the case of LIGO I, instead, we obtain $h_{c}=5.46 \times 10^{-22}\left(M / M_{\odot}\right)$ at $f_{c}=531 \mathrm{~Hz}$. In both cases, the signal-to-noise ratio is $S / N \sim 0.25$, but this can grow to be $\lesssim 4$ in the case of LIGO II. These ratios could be increased considerably with the detection of the black hole ringing following the initial burst [8]. Computing the emitted power as

$$
\frac{d E}{d t}=\frac{1}{32 \pi} \sum_{\ell, m}\left(\left|\frac{d Q_{\ell m}^{+}}{d t}\right|^{2}+\left|Q_{\ell m}^{\times}\right|^{2}\right),
$$

the total energy lost to gravitational radiation is $E=1.45 \times$ $10^{-6}\left(M / M_{\odot}\right)$. This is about two orders of magnitude smaller than the estimate made in [1] for a star with $a=0.54$, but larger than the energy losses computed recently in the collapse of rotating stellar cores to protoneutron stars [20].

In conclusion, we have presented the first waveforms from the gravitational collapse of rapidly rotating stars to black holes using 3D grids with Cartesian coordinates. The great potential shown by the PMR techniques employed here opens the way to a number of applications that would be otherwise intractable with uniform Cartesian grids. Work is in progress to consider initial models with realistic EOSs or in differential rotation, for which values $a \gtrsim 1$ can be reached and more intense gravitational radiation is expected.

We thank V. Ferrari, K. Kokkotas, N. Stergioulas, O. Zan-

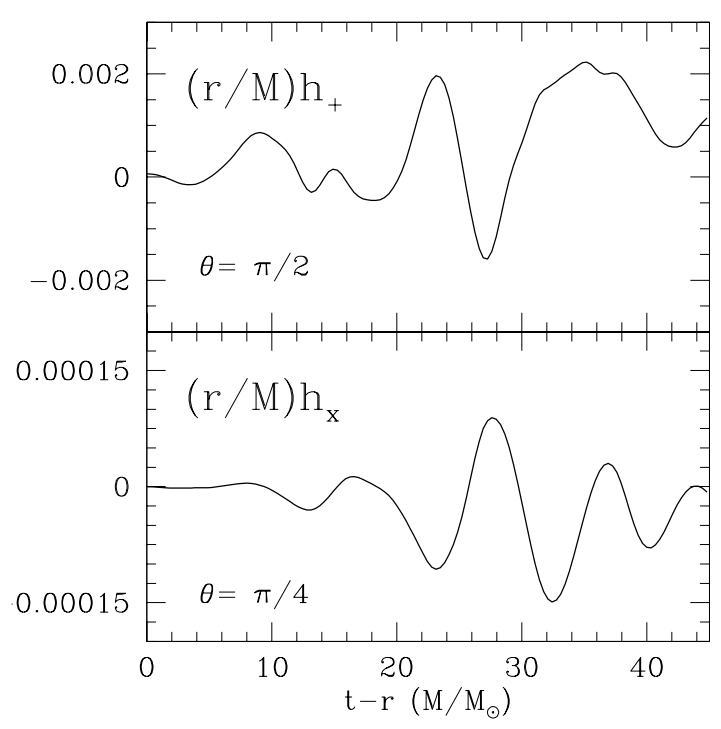

FIG. 3: TT gravitational-wave amplitudes extracted by the detector at $r_{\mathrm{ex}}=37.1 \mathrm{M}$ and for two different inclination angles.

otti for discussions, and C. Ott and B. Zink for help with the PMR algorithm. The computations were performed on $\mathrm{Al}$ bert100 at the University of Parma and Peyote at the Albert Einstein Institute. ES was supported by the DFG's SFB TR/7.

[1] R.F. Stark, T. Piran, Phys. Rev. Lett. 55, 891 (1985); Proceedings of the workshop Dynamical spacetimes and numerical relativity, Philadelphia, CUP, Cambridge UK, pp. 40-73 (1986)

[2] M. Shibata, T.W. Baumgarte, S.L. Shapiro, Phys. Rev. D 61, 044012 (2000)

[3] M. Shibata, Phys. Rev. D 67, 024033 (2003); Astrophys. J. 595, $992(2003)$

[4] M.D. Duez, S.L. Shapiro, H.J. Yo, Phys. Rev. D 69, 104016 (2004)

[5] L. Baiotti et al., Phys. Rev. D, in press, gr-qc/0403029

[6] M.J. Berger, J. Oliger, J. Comput. Phys. 53, 484 (1984)

[7] E. Schnetter, S.H. Hawley, I. Hawke, Class. Quantum Grav. 21, 1465 (2004)

[8] L. Baiotti et al., in preparation (2005)

[9] M.E. Rupright, A.M. Abrahams, L. Rezzolla, Phys. Rev. D 58, 044005 (1998)

[10] K. Camarda, E. Seidel, Phys. Rev. D 59, 064019 (1999)

[11] G. Allen, K. Camarda, E. Seidel, gr-qc/9806036(1998)

[12] V. Moncrief, Annals of Physics 88, 323 (1974)

[13] L. Rezzolla et al., Phys. Rev. D 59, 064001 (1999)

[14] J. Baker et al., Phys. Rev. D 62, 127701 (2000)

[15] J.A. Font et al., Phys. Rev. D 65, 084024 (2002)

[16] E.W. Leaver, Proc. R. Soc. Lond. A 402, 285 (1985)

[17] K.D. Kokkotas, B.G. Schmidt, Living Rev. Relativity 2 (1999)

[18] The frequencies for the QNMs of the black hole and for the $w$ modes of the star are those most easily obtained from tabulated values in the literature and serve here as indicative references.

[19] K. S. Thorne in 300 Years of Gravitation, S. W. Hawking, W. Israel, eds., CUP, Cambridge, UK (1987)

[20] E. Müller et al., Astrophys. J. 603, 221 (2004) 